\title{
MAGNETIC ANISOTROPY OF NiMn/Ni AND NiMn/Cu/Ni THIN FILMS
}

\author{
T. OgaWA and T. Sato
}

Department of Instrumentation Engineering, Faculty of Science and Technology Keio University, 3-14-1 Hiyoshi, Kohoku-ku, Yokohama, Kanagawa 223-8522, Japan

The magnetic anisotropy of reentrant spin glass NiMn this film, in the vicinity of the ferromagnetic $\mathrm{Ni}$ film, is investigated using $\mathrm{NiMn} / \mathrm{Ni}$, $\mathrm{Cu} / \mathrm{Ni} / \mathrm{Cu}(t=10 \AA$ and $500 \AA) / \mathrm{NiMn}$ and $\mathrm{NiMn} / \mathrm{Ni} / \mathrm{Cu}(t=10 \div$ $5000 \AA) / \mathrm{Ni}$ films. The unidirectional anisotropy field of NiMn layer in $\mathrm{NiMn} / \mathrm{Ni}$ film is smaller than that of bulk NiMn. In $\mathrm{Cu} / \mathrm{Ni} / \mathrm{Cu}(t=10 \AA$ and $500 \AA) / \mathrm{NiMn}$ film, the unidirectional anisotropy field is almost independent of the $\mathrm{Cu}$ spacer thickness. These results can be explained in terms of magnetic coupling between the magnetic layers in addition to the film structure of NiMn layer.

PACS numbers: $75.30 . \mathrm{Gw}, 75.50 . \mathrm{Lk}, 75.70 . \mathrm{Ak}$

\section{Introduction}

Bulk $\mathrm{Ni}_{100-x} \mathrm{Mn}_{x}$ disordered alloy exhibits a spin glass (SG) transition for $x>24$ and a reentrant spin glass (RSG) transition of paramagnetic to ferromagnetic to spin glass phase for $x<24$ [1]. Previously, we measured the magnetoresistivity of the $\mathrm{Ni}_{100-x} \mathrm{Mn}_{x}$ film with $x=27$ in NiMn/Ni bilayer film and found the enhancement of unidirectional anisotropy compared with that of bulk sample [2]. This change has been interpreted in terms of the proximity effect of spin glass and ferromagnetism. On the other hand, there has been no information about the magnetic anisotropy in the RSG NiMn film, having a lower Mn concentration, in the vicinity of the ferromagnetic Ni film. In addition, the correlation between the anisotropy in spin glass film and the film structure has rarely been studied. Thus, we performed the magnetic measurements of RSG-NiMn/Ni, $\mathrm{Cu} / \mathrm{Ni} / \mathrm{Cu} / \mathrm{RSG}-\mathrm{NiMn}$ and RSG-NiMn/Cu/Ni films with a various thickness of the $\mathrm{Cu}$ spacer layer, in addition to the evaluation of the film structure using atomic force microscopy (AFM). We discuss the anisotropy in a RSG-NiMn layer in terms of the contribution from the neighbor ferromagnetic layer and the film structure. 


\section{Experimental procedure}

Film deposition was performed on a quartz substrate at room temperature by the Ar ion-beam sputtering in an ultra-high vacuum chamber (base pressure: $\sim 10^{-9}$ Torr). The following samples were prepared: quartz/NiMn $500 \AA$, quartz/Cu $500 \AA / \mathrm{Ni} 80 \AA$, quartz/NiMn $500 \AA / \mathrm{Ni} 80 \AA$, quartz/NiMn $500 \AA$ / Cu $t \AA(t=10 \div 5000 \AA) / \mathrm{Ni} 80 \AA$ and quartz/Cu $500 \AA / \mathrm{Ni} 80 \AA / \mathrm{Cu} t \AA(t=10 \AA$ and $500 \AA) / \mathrm{NiMn} 500 \AA$. The deposition rates of $\mathrm{NiMn}, \mathrm{Ni}$, and $\mathrm{Cu}$ were $0.2 \AA / \mathrm{sec}$, $0.2 \AA / \mathrm{sec}$, and $0.6 \AA / \mathrm{sec}$, respectively. A Cu capping layer of $1000 \AA$ was deposited on all the samples to prevent oxidization of magnetic layer. The magnetization curves of $\mathrm{NiMn}, \mathrm{Cu} / \mathrm{Ni}, \mathrm{NiMn} / \mathrm{Ni}, \mathrm{NiMn} / \mathrm{Cu} / \mathrm{Ni}$, and $\mathrm{Cu} / \mathrm{Ni} / \mathrm{Cu} / \mathrm{NiMn}$ were obtained at $6 \mathrm{~K}$ after a field cooled procedure to evaluate the unidirectional anisotropy field of NiMn and the coercivity of $\mathrm{Ni}$. The magnetic measurements were performed using a superconducting quantum interference device (SQUID) magnetometer in a field applied parallel to the film surface. The AFM observation was performed in the atmosphere at room temperature.

\section{Results and discussion}

Figure 1 shows the magnetization curves of quartz/NiMn and quartz/Cu/Ni, where the unidirectional anisotropy field of NiMn and the coercivity of $\mathrm{Ni}$ are $320 \mathrm{Oe}$ and $120 \mathrm{Oe}$, respectively. The magnetization curve, obtained from the sum of those of NiMn and Ni, is shown also in Fig. 1 (denoted by NiMn+Ni). This type of hysteresis, expected when the magnetization of two layers behaves independently from each other, has the following characteristics: (1) a rapid change in magnetization approximately at $-100 \mathrm{Oe}$, corresponding to the reversal of magnetization of $\mathrm{Ni}$ film, and (2) a reversal of total magnetization appearing at a field lower than that of the quartz/NiMn film.

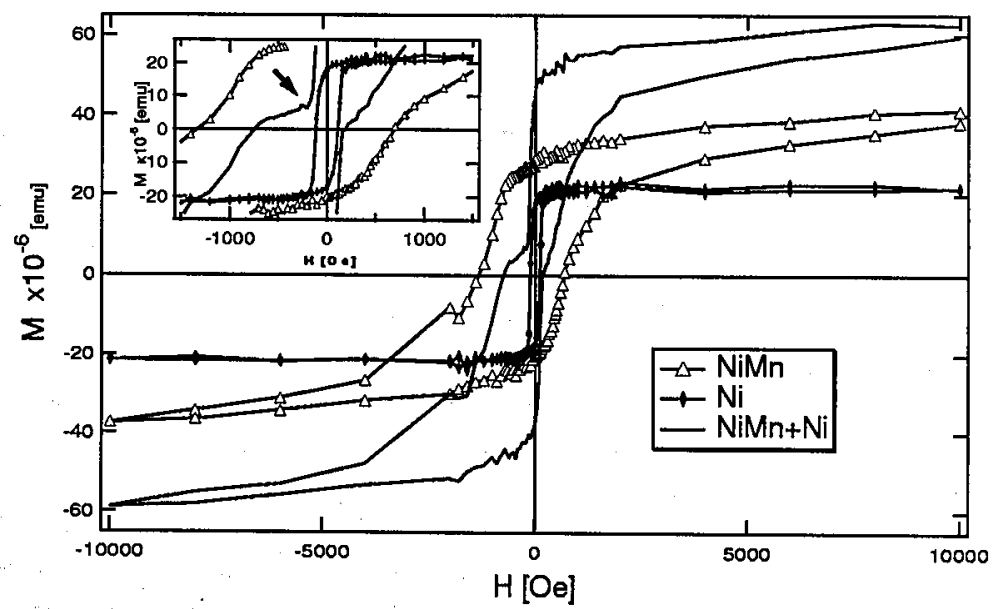

Fig. 1. The magnetization curves of quartz/NiMn, quartz/Cu/Ni, and $\mathrm{NiMn}+\mathrm{Ni}$ films. The inset shows the expanded area of a small applied field. The rapid change in magnetic moment in NiMn+Ni is shown by an arrow. 


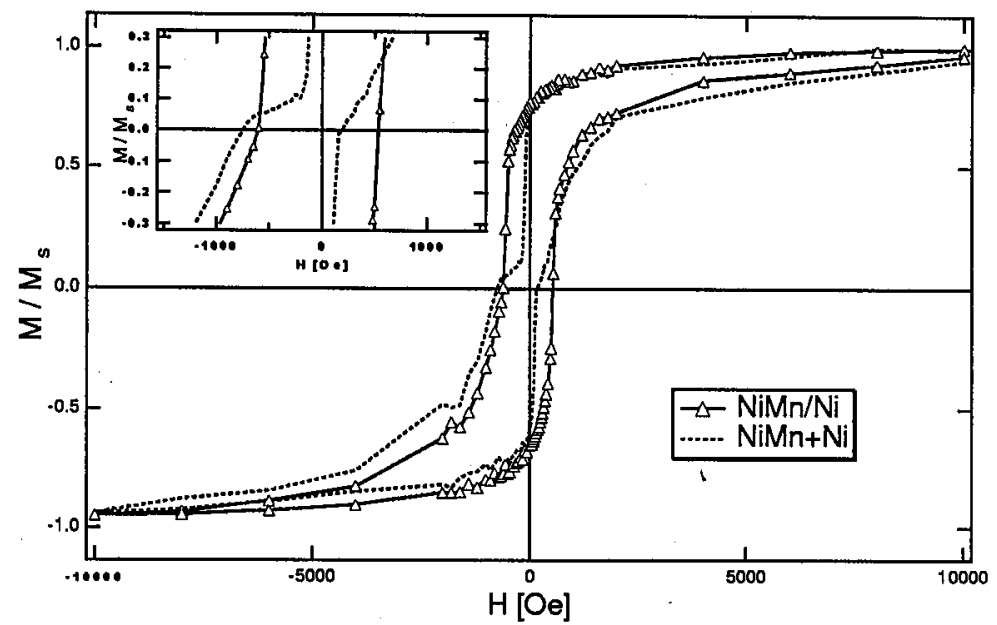

Fig. 2. The normalized magnetization curves of quartz/NiMn/Ni and NiMn+Ni films. The inset shows the expanded area of a small applied field.

Figure 2 shows the normalized magnetization curve of quartz/NiMn $/ \mathrm{Ni}$ film, in which the rapid change in magnetization, attributed to the reversal of magnetization in Ni layer, cannot be observed. Thus, this type of hysteresis suggests that the reversal of magnetization in the two magnetic layers occurs at a same applied field. In other words, the magnetic moments of two magnetic layers are strongly coupled. This can be explained in terms of an exchange coupling appearing at an interface between them. Based on this magnetization curve, we have found that the unidirectional anisotropy field in NiMn layer is about $60 \mathrm{Oe}$. This value corresponds to $20 \%$ of that in quartz/NiMn.

Figure 3 shows the normalized magnetization curves of quartz/Cu/Ni/ $\mathrm{Cu} t \AA / \mathrm{NiMn}$ films. In the hysteresis for $t=500 \AA$, we observe the rapid change in magnetization at \pm 100 Oe which is attributed to the reverse of the magnetization in Ni layer. In addition, the evaluated unidirectional anisotropy field in NiMn layer is the same as that of quartz/NiMn film. This indicates that the magnetization in NiMn and Ni layers behaves independently. The hysteresis of the sample with the thinnest Cu layer $(t=10 \AA)$ has the same characteristics as that in the sample with a thicker $\mathrm{Cu}$ layer. This indicates that the independent behavior of two magnetic layers essentially is preserved even at $t=10 \AA$. The detailed analysis of the hysteresis loop for $t=10 \AA$, however, shows the following features which are different from the thicker sample: (1) the decrease in unidirectional anisotropy field (260 Oe) in NiMn layer and (2) the three times enhancement of coercivity in $\mathrm{Ni}$ layer. This kind of difference can be explained in terms of two mechanisms, i.e., the weakly magnetic coupling appearing between the two magnetic layers and the difference of film structure related to the different thicknesses of Cu layers. The same characteristics were also observed in the quartz/NiMn/Cu/Ni film. Furthermore, the AFM observation of quartz/Cu/Ni/Cu/NiMn and quartz/NiMn/Cu/Ni films showed that the smooth and homogeneous surface is realized only in the former film. As a result, the characteristic change in magnetic anisotropy, observed 


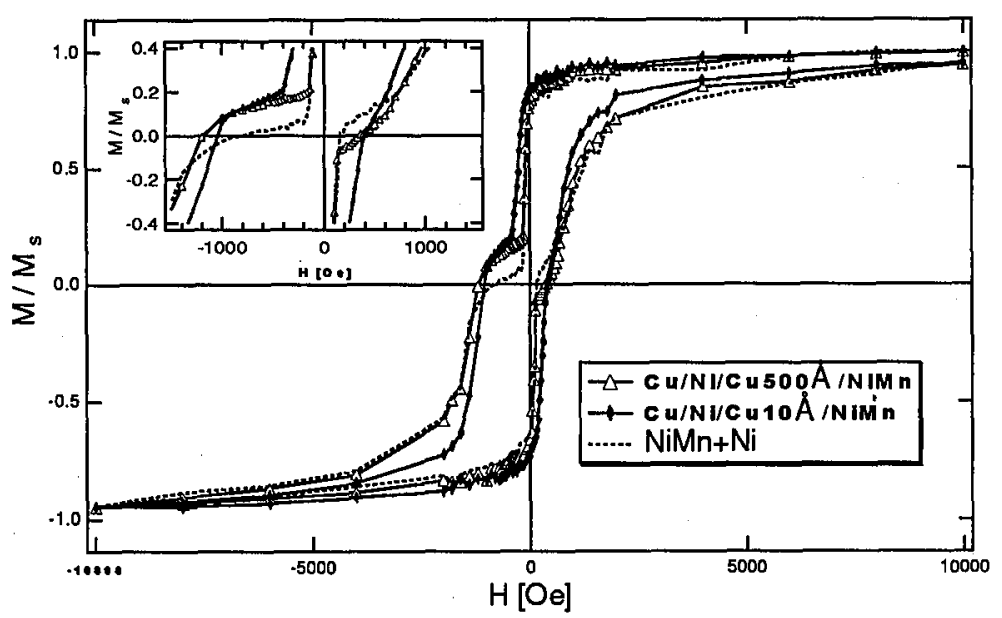

Fig. 3. The normalized magnetization curves of quartz/Cu/Ni/Cu $10 \AA / \mathrm{NiMn}$, quartz $/ \mathrm{Cu} / \mathrm{Ni} / \mathrm{Cu} 500 \AA / \mathrm{NiMn}$ and $\mathrm{NiMn}+\mathrm{Ni}$ films. The inset shows the expanded area of a small applied field.

in the sample with a thin $\mathrm{Cu}$ layer, should be partly explained in terms of the film structure.

Finally, we should note that there is a difference of change in the anisotropy in SG-NiMn and RSG-NiMn layers in the vicinity of the Ni layer. This may correlate with the long range ferromagnetic correlation existing only in the low temperature phase of RSG-NiMn.

\section{Conclusions}

The unidirectional anisotropy of RSG-NiMn layer, in the vicinity of Ni film, is smaller than that of bulk NiMn, which is opposite to that of SG-NiMn layer. This change is essentially attributed to the magnetic coupling between two magnetic layers. In addition, we found that the film structure plays an important role to the stability of unidirectional anisotropy in RSG-NiMn.

\section{References}

[1] W. Abdul-Razzaq, J.S. Kouvel, Phys. Rev. B 35, 1764 (1987).

[2] T. Ando, T. Sato, E. Ohta, J. Magn. Magn. Mater. 113, 115 (1992). 